\title{
Biomolecular interactions control the shape of stains from drying droplets of complex fluids
}

\author{
Cedric Hurth ${ }^{1,2^{*}}$, Rajneesh Bhardwaj ${ }^{3}$, Sahar Andalib ${ }^{4}$, Christophe Frankiewicz ${ }^{4}$, Andrew \\ Dobos $^{1,2}$, Daniel Attinger ${ }^{4}$, and Frederic Zenhausern ${ }^{1,2}$ \\ ${ }^{1}$ Center for Applied Nanobioscience\& Medicine, University of Arizona College of Medicine, Phoenix, AZ \\ 85004, USA \\ ${ }^{2}$ Translational Genomics Research Institute, Phoenix, AZ 85004, USA \\ ${ }^{3}$ Department of Mechanical Engineering, Indian Institute of Technology Bombay, Mumbai 400076, India \\ ${ }^{4}$ Department of Mechanical Engineering, Iowa State University, Ames, IA50011, USA
}

\begin{abstract}
When a sessile droplet of a complex fluid dries, a stain forms on the solid surface. The structure and pattern of the stain can be used to detect the presence of a specific chemical compound in the sessile droplet. In the present work, we investigate what parameters of the stain or its formation can be used to characterize the specific interaction between an aqueous dispersion of beads and its receptor immobilized on the surface. We use the biotin-streptavidin system as an experimental model. Clear dissimilarities were observed in the drying sequences on streptavidin-coated substrates of droplets of aqueous solutions containing biotin-coated or streptavidin-coated beads. Fluorescent beads are used in order to visualize the fluid flow field. We show differences in the distribution of the particles on the surface depending on biomolecular interactions between beads and the solid surface. A mechanistic model is proposed to explain the different patterns obtained during drying. The model describes that the beads are left behind the receding wetting line rather than pulled towards the drop center if the biological binding force is comparable to the surface tension of the receding wetting line. Other forces such as the viscous drag, van der Waals forces, and solid-solid friction forces are found negligible. Simple microfluidics experiments are performed to further illustrate the difference in behavior where is adhesion or friction are present between the bead and substrate due to the biological force. The results of the model are in agreement with the experimental observations which provide insight and design capabilities. A better understanding of the effects of the droplet-surface interaction on the drying mechanism is a crucial first step before the
\end{abstract}

\footnotetext{
*Corresponding author: churth@tgen.org | Alternate: fzenhaus@email.arizona.edu
} 
identification of drying patterns can be promisingly applied to areas such as immunology and biomarker detection. 


\section{Introduction}

Understanding how a drop of complex fluid dries on a solid surface and leaves a signature stain has various applications, ranging from manufacturing to medical diagnostic and forensic identification (1)-(3). Recent studies have focused on transport processes such as the pinning of a wetting line (4),(5), the effect of the solute particle size on the drying process (4), local velocity profiles (6), and evaporation fluxes at the droplet surface (7). A few studies have been centered on the influence of surface properties on drying process (8)-(14), but never concentrated on the role of biomolecular interaction between the solute and the substrate. A molecular recognition event is different from nonspecific van-der-Waals and electrostatic interactions studied so far. Such selectively sticky behavior is a powerful tool to differentiate between beads of different surface chemistry, as shown in the adhesion-based cell-separation microfluidic (15).

In the current study, we investigate how the drying process of a nanoliter droplet is affected by molecular interactions between the dispersed particles and a chemically modified surface with specific selective receptor. The biotin-streptavidin system was selected for its known strong non-covalent protein-cofactor interaction (16). Aqueous dispersions of biotin- or streptavidin-coated fluorescent particles are used to track the flow lines and visualize the effect of the streptavidin-coated surface on drying sequence. Our approach is analog to a recent study (17) but does not use external perturbations to force the drying process, which facilitates the development of future applications. 


\section{Experimental Methods}

Streptavidin- or biotin-coated fluorescent polystyrene (PS) particles (diam. 0.4-0.5 $\mu \mathrm{m})$ were ordered from Spherotec (Lake Forrest, IL) and diluted 500 or 1000 times in volume with ultrapure biotechnology performance certified (BPC) water. Streptavidincoated glass slides (Product Code SMS, Arrayit Corporation, Sunnyvale, CA) were ultrasonicated in ultrapure BPC water for 2 min, then rinsed and blown dry using filtered nitrogen. The $0.1 \mu \mathrm{L}$ droplets were deposited at room temperature of $22-24{ }^{\circ} \mathrm{C}$ with a relative humidity $(\mathrm{RH})$ of $20-24 \%$ using a micropipette. Fluorescence images were recorded on a Nikon Eclipse Ti-U inverted microscope with a 10X objective (Modulation Optics, Inc. Plan Fluor ELWD, NA=0.30), a mercury light source, and an amplified EMCCD (Andor Technology iXon, South Windsor, CT) cooled to $-20{ }^{\circ} \mathrm{C}$ using a $500 \mathrm{~ms}$ exposure. Both of biotin- and streptavidin-coated beads have different dyes embedded in their core; therefore the streptavidin surface was simultaneously imaged using fluorescein isothiocyanate (FITC) and $\mathrm{Cy} 3$ filters.

\section{Results and Discussion}

The evolution of the fluorescent particles coated with either biotin or streptavidin in the microdroplet is visualized on a glass surface coated with streptavidin. Figure 1 demonstrates that, in both cases, the particles first gather into forming a large peripheral ring. This indicates strong contribution from radial flow and that the drying process is driven by evaporation at the wetting line (20),(21). As the water evaporates, particles experience Brownian motion and the ring diameter stays constant. The two systems 
behave differently in the final stage. Whereas most streptavidin-coated beads are deposited in the central region of the droplet residue with fewer particles in the peripheral ring, the biotin-coated particles are distributed more uniformly (Figure 1(a)-(b)). The same observation can be drawn by comparing the radial particle distribution profile in a 90-degree quadrant area centered at the geometrical midpoint of the droplet and with the radius of the external peripheral ring (Figures 1(c)-(d)). About $30 \mathrm{~s}$ after the deposition, the distribution profiles show uniformly distributed beads around the center for both biotin- and streptavidin-coated solutions. However, the biotin-coated beads will retain a uniform angular distribution whereas the streptavidin-coated beads are concentrated within the first 35 microns. Here, we make the case that the different outcome is due to the existence of biomolecular force that resists the wetting force of the receding wetting line.

In order to understand how biomolecular interactions can prevent beads, which are attached to a solid-liquid interface, from sliding along with a moving fluid, we first need to consider the balance of the forces acting on a bead. We formulate therefore a mechanistic analytical model for one bead near the wetting line of the evaporating droplet. As shown in the force diagram (Figure 2(b)), the forces acting on the beads are the hydrodynamic drag force, a biological bonding force, a friction force, a Van der Waals force, and a surface tension force.

To evaluate the drag force caused by the moving fluid with the binding forces, we assembled a Hele-Shaw flow cell out of polydimethylsiloxane (PDMS, Sylgard 184, Dow Corning, Midland, MI). By flowing water at increasing flow rates after biotin and streptavidin beads have been deposited on the bottom surface (streptavidin-coated glass 
slide), we can increase the drag force on the bead and qualitatively compare it to the resisting forces (van der Waals and friction forces for both types of beads together with a biological bonding force, only for biotin-coated beads). The flow channel is rectangular $(0.5 \times 2 \times 10 \mathrm{~mm})$. Using syringe pumps, the pressure drop $\Delta p$ along the channel is varied and the presence of beads on the glass slide is monitored by fluorescence. The PDMS flow cell was first flushed with a 1:500 dilution of biotin-coated beads at a low flow rate $(0.2-0.5 \mathrm{~mL} / \mathrm{min})$. The beads were then allowed to settle in the dark for $30 \mathrm{~min}$, before a 1:500 dilution of streptavidin-coated beads was flowed through the cell at an equally slow flow rate. The cell was left to rest in the dark for another $30 \mathrm{~min}$. The flow cell was then flushed with water at increasing flow rates of $0.5,1,2,5,10$, and $14 \mathrm{~mL} / \mathrm{min}$. The number of beads of each type is counted using image processing software (ImageJ, NIH, Bethesda, MD) by thresholding the grayscale image and performing particle analysis. The selection criteria were particle sizes and a minimum circularity (or roundness) of 0.8 . The number of streptavidin beads present on the surface for the lowest rinsing flow rate tested is much smaller than the number of biotin beads due to higher affinity of the biotin beads for the surface. When the rinsing flow rate is increased from 0.5 to $1.0 \mathrm{~mL} / \mathrm{min}$, most of the streptavidin beads are flushed away from the surface whereas the high number of biotin beads on the surface remains unchanged (Supplementary Figure S1)Error! Reference source not found.. Table I gives the number of particles after the deposition step and for each flow rates of rinsing water, as well as the drag force. The results in Table 1 confirm that a hydrodynamic drag force of $0.2-0.8 \mathrm{pN}$ is sufficient to wash out the streptavidin beads which are not attached by biomolecular forces, while the 
biotin beads remain attached to the surface even for a drag force one order of magnitude larger due to the presence of biomolecular interactions.

In the Stokes flow conditions, which are typical for drop drying, the drag force on a bead attached to the solid-liquid interface is estimated at a distance $z$ with Faxen's law (22) to:

$$
F_{d r a g}=6 \pi \mu R v_{\text {rad }} /\left(1-\frac{9}{16}\left(\frac{R}{z}\right)+\frac{1}{8}\left(\frac{R}{z}\right)^{3}\right),
$$

where $\mu$ is the dynamic viscosity of the droplet liquid, $R$ is the radius of the bead, and $v_{\text {rad }}$ is the radial velocity inside the droplet due to the evaporation. The radial velocity $v_{\text {rad }}$ scales as $j / \rho$, where $j$ is evaporative flux $\left[\mathrm{kg} \mathrm{m}^{-2} \mathrm{~s}^{-1}\right]$ and $\rho$ is density of the liquid (21).

Table I. Number of beads present on the surface after deposition and after each flow rate of rinsing water tested. The drag force is calculated by Eqn. (1).

\begin{tabular}{|c|c|c|c|c|c|c|c|}
\hline \multirow{2}{*}{\multicolumn{2}{|c|}{$\begin{array}{c}\text { flow rate }[\mathrm{mL} / \mathrm{min}] \\
\text { drag force }(\mathrm{pN})\end{array}$}} & 0.5 & 1.0 & 2.0 & 5.0 & 10.0 & 14.0 \\
\hline & & 0.20 & 0.40 & 0.81 & 2.0 & 4.0 & 5.7 \\
\hline & deposited & \multicolumn{6}{|c|}{ Number of beads on streptavidin surface } \\
\hline streptavidin & 207 & 5 & 2 & 1 & 1 & 1 & 1 \\
\hline biotin & 138 & 27 & 26 & 25 & 25 & 25 & 25 \\
\hline
\end{tabular}

The bonding force involves the biological and van der Waals interaction between the biotin-coated bead and the streptavidin-coated substrate. The biological force is modeled by assuming that each bond between the ligand covalently bound to the bead and the receptor on the substrate exerts either a radial outward (from the center of the bead) tensile force or no force at all, i.e. bonds cannot exert a compression force (23). To quantify the force, we consider a spherical bead attached to the surface by a biological bond. We estimate the maximum bond angle $\theta$, as a function of bond length $l$ and the 
bead radius $R$ (24)Error! Reference source not found.. The relation between $R, \theta$, and $l$ (Figure 2(a)) is expressed as:

$R(1-\cos \theta)=l \cos \theta$

The surface area $A$ of the bead that is connected to the substrate by the biological bond is obtained by the following integration:

$A=\iiint r^{2} \sin \theta d r d \phi d \theta=2 \pi R^{2} \int_{0}^{\theta} \sin \theta d \theta=2 \pi R^{2}(-\cos \theta)_{0}^{\theta}=2 \pi R^{2}(1-\cos \theta)$

For small values of $\theta$, i.e. for the typical situation of a molecular bond much smaller than the bead, $l<<R$, we can simplify the above equations into:

$\theta \cong \sqrt{\frac{2 l}{R}} \quad$, and $A=2 \pi \mathrm{R} l$

For biomolecules shorter than the bead radius, the biological force is given as the product of the attractive force per bond and the number $\mathrm{A}^{\cdot} \rho_{\text {bio }}$; of bonds involved in the interaction:

$F_{\text {bonding }} \approx 2 \pi R l f \rho_{\text {bio }}$

In the above equation, $f$ is the attractive force per bond. As reported in Table II there is a large uncertainty on the force $f$, because of the experimental methods used, e.g. stretching bonds with the tip of an atomic force microscope (19),(25),(26), by micropipette aspiration (27)(27), or by optical tweezers (28).

We evaluate the van der Waals force (29) at a distance $l$, i.e. the typical distance between the bead surface and the streptavidin-coated surface. In all cases, the distance between the bead surface and either biotin or streptavidin is set by the linker chain (manufacturer communication: $\mathrm{C} 11, \approx 1.7 \AA$ ), and the coated molecule. The biotin molecule maximum 
internuclear distance is $\approx 1.4 \AA$ whereas the streptavidin protein has a typical dimension of $4.7 \mathrm{~nm}$ (18). For the streptavidin-coated surface, the distance between the glass surface and the streptavidin coated is set by the size of the streptavidin as a smaller linker is used. This gives $l \approx 0.17+4.7+4.7=9.6 \mathrm{~nm}$ as the minimum distance for streptavidin-coated beads and the streptavidin-coated surface, and $l \approx 0.17+0.14+4.7=5.0 \mathrm{~nm}$ for the biotincoated beads and the streptavidin surface. The resulting van der Waals force is:

$F_{V d W}=\frac{1}{96} H_{w a t} R^{3} \frac{\alpha_{r d d}}{l^{2}(l+2 R)^{2}}$

with $H_{\text {wal }}=210^{-20} \mathrm{~J}$, the Hamacker constant for water and $\alpha_{r t d}$, a retardation factor depending on the distance between the bead and surface (29).

In the case of streptavidin-coated beads, there is no biological bonding with the surface, and the only cohesive force is the van der Waals force between the particle and the surface. We assume that the electrostatic contribution is largely repulsive since both the bead and the surface are coated with a similar number of protein molecules in a similar charge state.

The surface tension force on the bead at the wetting line in the two cases scales as $\gamma R$, where $\gamma$ and $R$ are the liquid-particle surface tension $\left[\mathrm{Nm}^{-1}\right]$ and radius of the bead $[\mathrm{m}]$, respectively.

The friction force $\left(F_{\text {friction }}\right)$ is estimated as its maximum value at a flow rate of $\mathrm{Q} \approx 0.5$ $\mathrm{mL} / \mathrm{min}$ when most streptavidin-coated beads are already washed off the surface (Table I).

The total forces on a bead in the radial directions (Figure 2(b)) are expressed as: 
$F_{r+}=F_{\text {drag }}+F_{\text {bonding }}+F_{\text {friction }}$

$F_{r-}=F_{\text {tension }}$

Note that the van der Waals force is absent in the above equations because we consider forces or force components in the radial direction only. The beads slide if $F_{r-}>F_{\mathrm{r}+}$. We can therefore describe the situation with a dimensionless number $S$ :

$S=\frac{F_{r+}}{F_{r-}}$

The beads slide for $S<<1$ and pin for $S \gg>1$. Intermediate values with $\mathrm{S}=\mathrm{O}(1)$ likely correspond to cases where a fraction of the beads pin while the other fraction slide. Since the van der Waals and drag force are orders of magnitude smaller than the surface tension and biological bonding forces, the sliding or pinning motion of the bead is simply controlled by the ratio of the bonding force and the surface tension, as follows:

$S=\frac{F_{\text {bonding }}}{\gamma R}=\frac{2 \pi \rho l f}{\gamma}$

The parameters used in the calculations as well as the magnitude of the forces on a single bead at the wetting line are given in Table II.

Table II. Values of the parameters used for calculating the bonding and drag force.

\begin{tabular}{|c|c|}
\hline Parameter & Value \\
\hline Bead radius, $R$ & $2.5 \times 10^{-7} \mathrm{~m}$ \\
\hline Biot./Strept. Attractive force per bond, $f$ & $150_{-45}^{+850} \times 10^{-12} \mathrm{~N}^{1}$ \\
\hline $\begin{array}{rr}\text { Bond length }^{2}, l & \begin{array}{r}\text { biotin } \\
\text { streptavidin }\end{array}\end{array}$ & $\begin{array}{l}5.0 \times 10^{-9} \mathrm{~m} \\
9.6 \times 10^{-9} \mathrm{~m}\end{array}$ \\
\hline $\begin{array}{r}\text { Bead receptor density }^{3} \\
\text { biotin } \\
\text { streptavidin }\end{array}$ & $\begin{array}{l}1.1 \times 10^{16} \text { molecules.m } m^{-2}\left( \pm 0.210^{16}\right) \\
3.1 \times 10^{16} \text { molecules.m } m^{-2}\left( \pm 0.610^{16}\right)\end{array}$ \\
\hline Dynamic viscosity of water, $\mu$ & $1.0 \times 10^{-3} \mathrm{~Pa}-\mathrm{s}$ \\
\hline $\begin{array}{r}\text { Threshold flow rate in channel, } Q \\
\text { biotin } \\
\text { streptavidin }\end{array}$ & $\begin{array}{l}>14 \mathrm{~mL} / \mathrm{min} \\
<0.5 \mathrm{~mL} / \mathrm{min}\end{array}$ \\
\hline
\end{tabular}




\begin{tabular}{|c|c|c|}
\hline Height of channel, $h$ & \multicolumn{2}{|c|}{$0.5 \mathrm{~mm}$} \\
\hline Width of channel, $w$ & \multicolumn{2}{|c|}{$2 \mathrm{~mm}$} \\
\hline Receding angle & \multicolumn{2}{|c|}{$\begin{array}{c}\text { Biotin beads } 26^{\circ} \\
\text { Streptavidin beads } 30^{\circ}\end{array}$} \\
\hline $\begin{array}{r}\text { Forces } \\
\text { Van der Waals } \\
\text { drag force } \\
\text { biological bonding force } \\
\text { surface force } \\
\text { friction } 4\end{array}$ & $\begin{array}{r}\text { biotin beads } \\
0.35 \times 10^{-15} \mathrm{~N} \\
1.44 \times 10^{-14} \mathrm{~N} \\
0.33 \pm 0.34 \times 10^{-9} \mathrm{~N} \\
1.8 \times 10^{-8} \mathrm{~N} \\
<2.44 \times 10^{-13} \mathrm{~N}\end{array}$ & $\begin{array}{r}\text { streptavidin beads } \\
0.10 \times 10^{-15} \mathrm{~N} \\
1.44 \times 10^{-14} \mathrm{~N} \\
0 \mathrm{~N} \\
1.8 \times 10^{-8} \mathrm{~N} \\
<2.44 \times 10^{-13} \mathrm{~N}\end{array}$ \\
\hline
\end{tabular}

1 From reference (19) and references therein.

2 C11 linker arm (manufacturer communication) and biotin-streptavidin bond length of $0.9 \mathrm{~nm}$ (19).

3 From the manufacturer data sheet. The error is estimated from standard errors on fluorescence measurements.

4 Calculated for a max. flow rate $\mathrm{Q}=0.5 \mathrm{~mL} / \mathrm{min}$ where most streptavidin beads are already washed away (Table I)

The sum of projected radial forces (Table II) in the two cases is plotted in Figure 2(c). For a dispersion of biotin-coated beads drying on a surface coated with streptavidin, there is a biological bonding force that resists the receding of a wetting line, leaving the original drop area covered with beads that have been left behind the receding wetting line. On the other hand, there is no biological bonding force for a dispersion of streptavidin-coated beads drying on a streptavidin-coated surface. Therefore, in this case, the beads will recede with the wetting line and accumulate in larger number at the center of the original drop area. In our calculations, the biological force is on the order $110^{-9} \mathrm{~N}$, i.e. 3 orders of magnitude larger than the drag force at the largest value of flow rate achieved in the flow cell experiments (Table I). The comparative analytical model allows explaining the two observations made showing different behavior depending on the bead coating. This explains the different aspects between stains 1(a) and 1(b) and illustrates the effect of biomolecular binding on the drying process of the droplet.

\section{Conclusion}

An experimental study has been performed to illustrate how the presence of a strong specific biomolecular interaction, e.g. biotin-streptavidin, between a solute and a surface 
can modify the drying process of a microdroplet. Fluorescent visualization of dilutions of fluorescent streptavidin- and biotin-coated polystyrene microparticles deposited on streptavidin-coated surfaces show a difference in the distribution of particles. As the wetting line recedes, the interaction with the streptavidin surface distributes the biotin particles more uniformly. Furthermore, shear flow measurements in a PDMS Hele-Shaw cell and an analytical model suggest that the additional adhesion force from the molecular interaction between biotin on the beads and streptavidin on the surface can overcome the drag force experienced by the beads in the flow cell. In the case of streptavidin beads, the drag force is much larger than that of the adhesive van der Waals force, as soon as the shear flow rate exceeds $1 \mathrm{~mL} / \mathrm{min}$ and the beads cannot stick to the surface. The effect of a biological interaction on the drying sequence reported here is a first step towards developing a method to detect and quantify a specific biomarker in a biological fluid when coupled with a pattern recognition algorithm previously developed (30).

\section{Acknowledgments}

The authors are grateful for helpful discussions with Dr. Jian Gu as well as the technical support of Matthew Barrett, both at the Center for Applied Nanobioscience \& Medicine. Funding from the National Science Foundation (Grant \# 1211187), from the IBIS foundation of Arizona, the Helios Foundation, as well as the University of Arizona College of Medicine Phoenix are acknowledged. 


\section{FIGURE CAPTIONS}

Fig. 1. Evolution of a $0.2 \mu \mathrm{L}$ droplet containing streptavidin- (a) and biotin-coated (b) beads on a streptavidin-coated glass surface. Fluorescence intensity of the radial distribution of beads in a quadrant centered on the midpoint of the droplet for streptavidin- (c) and biotin-coated (d) beads solutions $30 \mathrm{~s}$ after deposition (dashed lines) and for the final frame (plain lines). Scale bar is $250 \mu \mathrm{m}$. More details are given in the text.

Fig. 2. (a) Geometry of the spherical bead attached to the surface. $A$ is the area of the bead that can be biologically bound to the slide. (b) Schematic of the forces acting on the bead near the wetting line. The surface tension force acting on one bead is the blue vector. (c) Comparison of forces in play for biotin- and streptavidin-coated beads (values in Table II). Per Eqns (9) and (10), $S \approx 2.8$ and $S \approx 1.510^{-5}$ for biotin- and streptavidincoated beads, respectively. These values explain the respective sticking and sliding properties. 


\section{FIGURE 1}
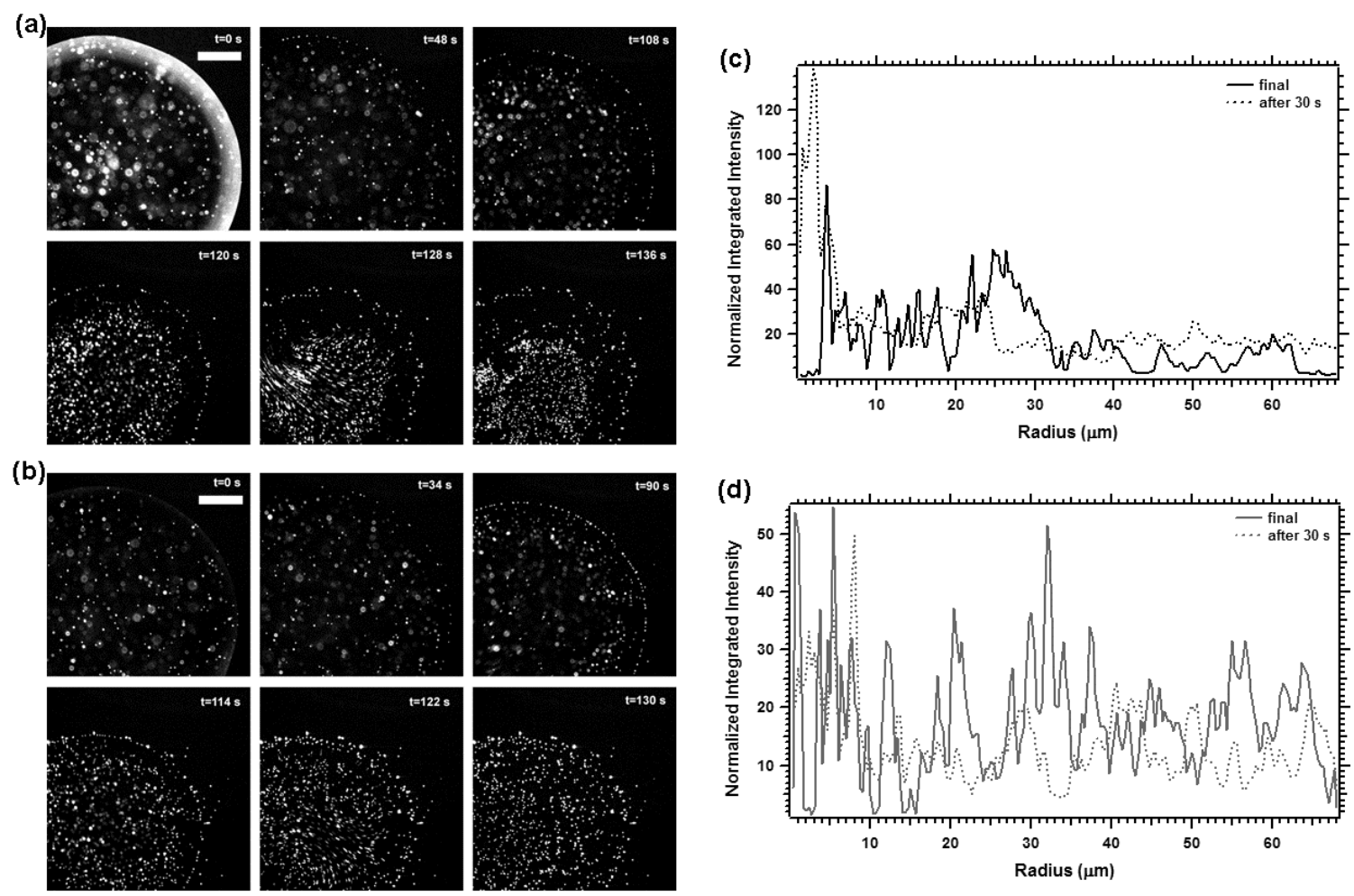
FIGURE 2

(a)

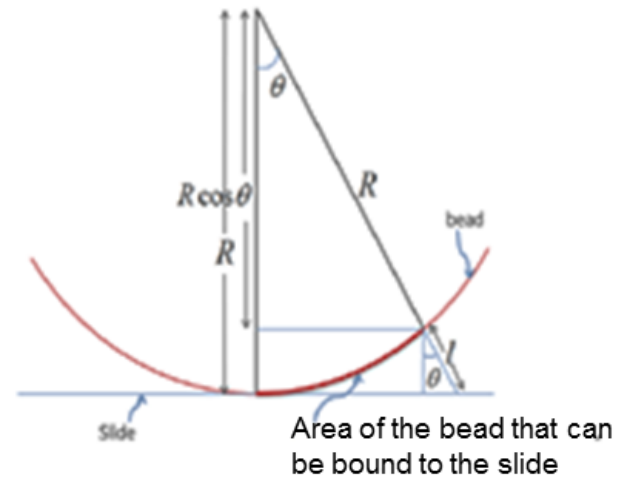

(b)
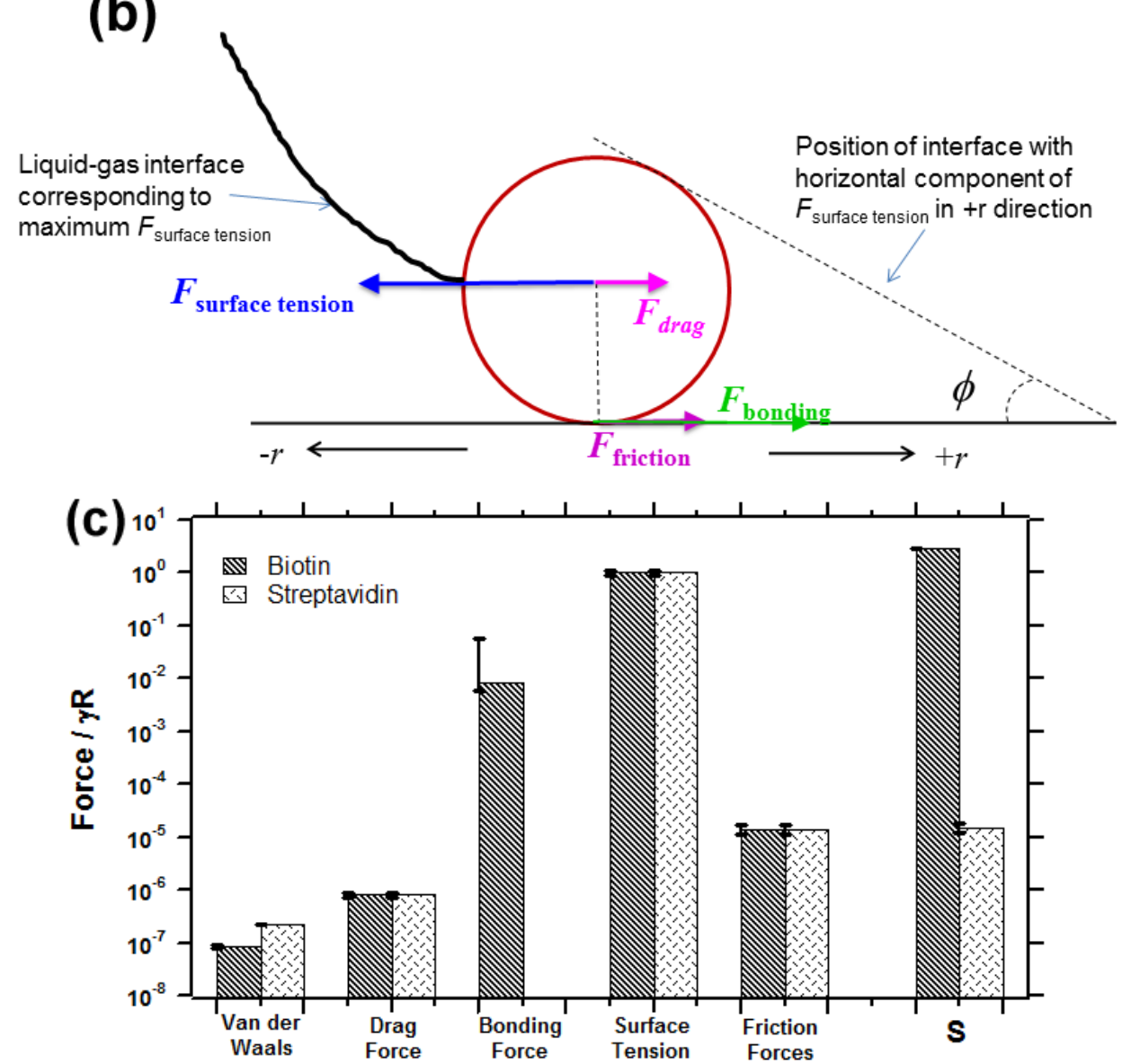


\section{REFERENCES}

(1) D. Maltoni, Handbook of fingerprint recognition, Springer-Verlag, New York, 2003.

(2) V. Dugas, J. Broutin and E. Souteyrand, Langmuir, 21 (2008) 9130.

(3) M. Maillard, L. Motte and M.P. Pileni, Adv. Mater., 13 (2001) 200.

(4) A.S. Sangani, C.H. Lu, K.H. Su and J.A. Schwarz, Phys. Rev. E, 80 (2009) 011603.

(5) U. Thiele, Adv. Coll. Interf. Sci., 206 (2014) 399.

(6) H. Hu and R.G. Larson, Langmuir, 21 (2005) 3963.

(7) H. Hu and R.G. Larson, J. Phys. Chem. B, 106 (2002) 1334.

(8) K. Sefiane and R. Bennacer, Adv. Colloid Interface Sci., 147-148 (2009) 263.

(9) K.F. Baughman, R.M. Maier, T.A. Norris, B.M. Beam, A. Mudalige, J.E. Pemberton and J.E. Curry, Langmuir, 26 (2010) 7293.

(10) W. Xu, R. Leeladhar, Y.T. Tsai, E.H. Yang and C.H. Choi, Appl. Phys. Lett., 98 (2011) 073101.

(11) A. Accardo, F. Gentile, F. Mecarini, F. De Angelis, M. Burghammer, E. Di Fabrizio and C. Riekel, Microelectron. Eng., 88 (2011) 1660.

(12) C.Y. Lee, B.J. Zhang, J. Park and K.J. Kim, Int. J. Heat Mass Tran., 55 (2012) 2151.

(13) W.D. Ristenpart, P.G. Kim, C. Domingues, J. Wan and H.A. Stone, Phys. Rev. Lett., 99 (2007) 234502.

(14) R.G. Larson, M.A. Lopez, D.W. Lim and J. Lahann, Mater. Res. Soc. Symp. Proc., 1273E (2010) MM03-01.

(15) J. Miwa, Y. Suzuki, and N. Kasagi, J. Microelectromech. Syst., 17 (2008) 611.

(16) A. Holmberg, O. Nord, M. Lukacs, J. Lundesberg and M. Uhlén, Electrophoresis, 26 (2005) 501.

(17) J.R. Trantum, D.W. Wright and F.R. Haselton, Langmuir, 28 (2012) 2187.

(18) C.S. Neish, I.L. Martin, R.M. Henderson and J.M. Edwardson, Br. J. Pharmacol., 135 (2002) 1943.

(19) T. Wong, A. Chilkoti and V.T. Moy, Biomol. Eng., 16 (1999) 45.

(20) R. Bhardwaj, X. Fang and D. Attinger, New J. Physics, 11 (2009) 075020.

(21) R. Bhardwaj, X. Fang, P. Somasundaran and D. Attinger, Langmuir, 26 (2010) 7833.

(22) J. Leach, H. Mushfique, S. Keen, R. Di Leonardo, G. Ruocco, J.M. Cooper and M.J. Padgett, Phys. Rev. E, 79 (2009) 026301.

(23) G.I. Bell, Science. 200 (1978) 618.

(24) A. Tozeren and K. Ley, Biophys J., 63 (1992) 700.

(25) G.U. Lee, D.A. Kidwell and R.J. Colton, Langmuir, 10 (1994) 354.

(26) M. de Odrowaz Piramowicz, P. Czuba, M. Tagosz, K. Burda and M. Szymonski, Acta Biochim. Pol., 53 (2006) 93.

(27) E. Evans, K. Ritchie and R. Merkel, Biophys. J, 68 (1995) 2580.

(28) A.L. Stout and W.W. Webb, Meth Cell Biol, 55 (1998) 99.

(29) C. Tien, Granular Filtration of Aerosols and Hydrosols, Butterworth, Boston, 1989.

(30) N. Kim, Z. Li, C. Hurth, F. Zenhausern, S.F. Chang and D. Attinger, Anal. Methods, 4 (2012) 50. 